\title{
Positional Therapy in a Patient With Refractory Treatment-Emergent Central Sleep Apnea
}

\author{
William Palmer, Miriam Jaziri, Maria Tovar \\ Department of Sleep Disorders, Henry Ford Hospital, Detroit, MI, USA
}

Received October 20, 2021

Revised December 4, 2021

Accepted December 15, 2021

Address for correspondence

Miriam Jaziri, MD

Henry Ford Hospital,

2799 W Grand Blvd., Detroit,

MI 48202, USA

Tel: $+1-313-932-0517$

Fax: +1-313-916-5150

E-mail: mjaziri1@hfhs.org
Treatment-emergent central sleep apnea (TE-CSA) is commonly encountered during the treatment for obstructive sleep apnea (OSA) with positive airway pressure (PAP) and usually remains self-limited. Persistent TE-CSA is sporadically seen with PAP therapy and has only rarely been described with hypoglossal nerve stimulation (HGNS). We report the case of a 60 -year-old female patient with moderate OSA that progressed to TE-CSA with PAP therapy. A prolonged trial with PAP therapy was limited because the patient experienced recurrent aerophagia and subsequently underwent HGNS implantation. HGNS titration led to improved control of the patient's OSA, but TE-CSA recurred and demonstrated a strong positional component. Lateral positional therapy was implemented with adequate control of respiratory events. TE-CSA can persist throughout different treatment modalities, including HGNS. The patient's successful lateral sleep therapy for persistent and positionally exacerbated TE-CSA demonstrates the benefit of a well-known sleep apnea treatment for this poorly understood condition.

J Sleep Med 2021;18(3):182-185

\section{INTRODUCTION}

Treatment-emergent central sleep apnea (TE-CSA) is commonly encountered in patients who have been treated with positive airway pressure (PAP) for obstructive sleep apnea (OSA). Most cases are self-limited and resolve over time or with adjustments in PAP therapy. Persistent TE-CSA is seen in only $0.9 \%-3 \%$ of all patients on PAP therapy and remains poorly understood. ${ }^{1}$ We present the case of a patient who developed TE-CSA that was refractory to conventional and advanced treatments, including hypoglossal nerve stimulation (HGNS), and responded well to a treatment that is sometimes overlooked.

\section{CASE REPORT}

A 60-year-old female with a medical history of hypertension, hyperlipidemia, arthritis, neuralgia, and depression was evaluated for snoring, witnessed apneas, frequent nighttime awakenings, and difficulty sleeping for the last 10 years. Her

This is an Open Access article distributed under the terms of the Creative Commons Attribution Non-Commercial License (https://creativecommons.org/licenses/by-nc/4.0) which permits unrestricted non-commercial use, distribution, and reproduction in any medium, provided the original work is properly cited.
Epworth sleepiness score (ESS) was high at 17. Her physical examination revealed an obese body habitus with a body mass index (BMI) of 31.17, normal mandibular position, normal external nose with midline nasal septum, normal hard and soft palate, normal size uvula, absent tonsils after tonsillectomy during childhood, Friedman class two tongue position, and an unremarkable neck circumference of $37 \mathrm{~cm}$. She only slept at night and had a regular sleep schedule. Her usual bedtime was 9:30 PM with a sleep onset latency of 20-30 minutes, and she was getting out of bed around 5:30 AM in the morning. During the night she had one to two night-time awakenings with difficulties falling back asleep which resulted in a diminished total sleep time of only four to five hours.

Her initial polysomnogram (PSG) showed moderate OSA with an overall apnea-hypopnea index (AHI) of 19.1 showing mainly obstructive events (only two central apneas). Respiratory events were almost equally seen in all sleep positions with a lateral AHI of 19.5 and a supine AHI of 16.7. Only about $15 \%$ of total sleep time was spent in the supine position (Fig. $1 \mathrm{~A}$ and Table 1).

She was subsequently treated with auto-adjusting continuous PAP (auto CPAP) 5-15 $\mathrm{cm} \mathrm{H}_{2} \mathrm{O}$ using a Resmed device (Resmed; US and Canada ResMed Corp, San Diego, CA, USA) 
and CPAP compliance was closely monitored remotely via "MyAir," the Resmed associated online cloud. She developed aerophagia within the first 30 days, so her interface was changed, and her CPAP setting was adjusted to a fixed pressure of CPAP $11 \mathrm{~cm} \mathrm{H}_{2} \mathrm{O}$ based on her 95th percentile pressure. On her twomonth follow up visit she continued to be compliant with CPAP therapy (60\%-70\% usage of CPAP for four or more hours per 24-hour period), but still experienced aerophagia despite switching to several different mask models, using a wedge pillow, and a trial of simethicone. A download from her CPAP device showed a total AHI of 6.4 with a central AHI of 5.6. A diagnosis of TE-CSA was considered and expected to improve over time, however, a prolonged CPAP trial was not feasible since the patient now also started having pressure intolerance, and it was decided to proceed with a BPAP titration.

During the first in-laboratory PAP titration, the patient was titrated on bilevel PAP (BPAP) with an inspiratory positive airway pressure (IPAP) from 10 to $18 \mathrm{~cm} \mathrm{H}_{2} \mathrm{O}$ and an expiratory
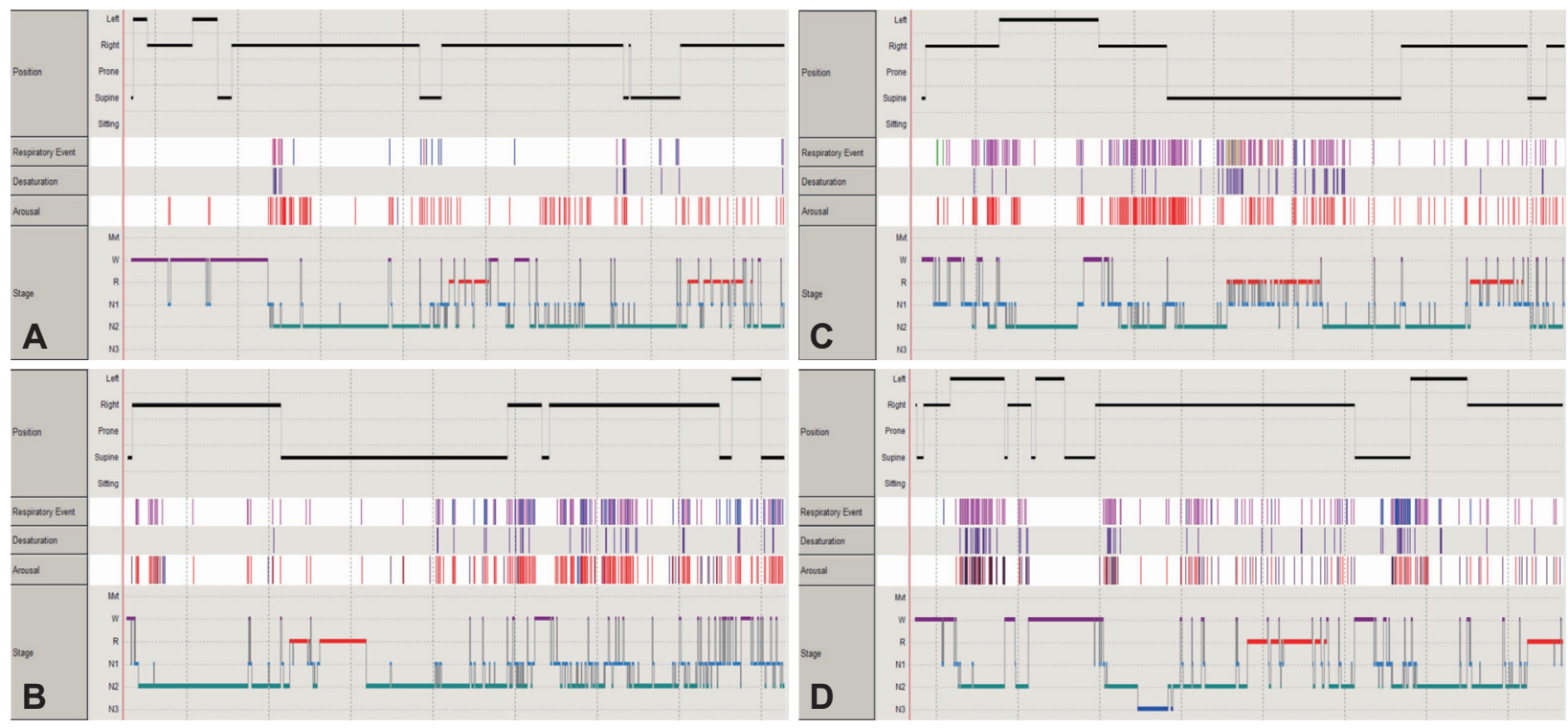

Figure 1. Hypnograms representing the patient's positional changes, oxygen desaturations, arousals, respiratory events, and sleep stages. For respiratory events, the pink lines indicate obstructive events and blue and green lines indicate central apneas and hypopneas respectively. A: Initial diagnostic polysomnogram showing undifferentiated obstructive sleep apnea that indicate no obvious preference for position or sleep phase. B: Bilevel positive airway pressure (BPAP) titration concerning for treatment-emergent central sleep apnea (TECSA) as seen by an increase of blue lines in the second half of the study. C: Titration with BPAP and adaptive servo-ventilation showing persistent TE-CSA and a slight trend of increased apneic events during supine sleep. D: *Inspire titration with a high number of central apneas in the supine position as seen by the abundance of blue lines while the patient is supine in the last third of the study after the patient had already shown good control of respiratory events in the lateral position during the middle third of the study. *inspire is a product of Inspire Medical Systems, Inc., Golden Valley, MN, USA.

Table 1. Differentiation of respiratory events during in-laboratory polysomnogram and titration as observed by position

\begin{tabular}{|c|c|c|c|c|c|c|c|c|}
\hline & \multicolumn{2}{|c|}{$\begin{array}{l}\text { Initial diagnostic } \\
\text { polysomnogram }\end{array}$} & \multicolumn{2}{|c|}{$\begin{array}{l}\text { First PAP titration } \\
\text { (BPAP) }\end{array}$} & \multicolumn{2}{|c|}{$\begin{array}{l}\text { Second PAP titration } \\
\text { (BPAP and ASV) }\end{array}$} & \multicolumn{2}{|c|}{ Inspire* titration } \\
\hline & Non-supine & Supine & Non-supine & Supine & Non-supine & Supine & Non-supine & Supine \\
\hline Sleep time (min) & 292.5 & 53.8 & 248 & 182.8 & 247.8 & 187.5 & 329.4 & 23.5 \\
\hline Obstructive apnea & 1 & 0 & 0 & 0 & 0 & 3 & 0 & 0 \\
\hline Mixed apnea & 0 & 0 & 0 & 0 & 0 & 11 & 0 & 0 \\
\hline Central apnea & 1 & 1 & 34 & 19 & 7 & 17 & 10 & 14 \\
\hline Hypopneas & 93 & 14 & 87 & 19 & 0 & 0 & 6 & 3 \\
\hline Central hypopneas & 0 & 0 & 0 & 0 & 89 & 67 & 0 & 0 \\
\hline Apnea-hypopnea index & 19.5 & 16.7 & 29.3 & 12.5 & 23.2 & 31.4 & 2.9 & 43.4 \\
\hline Obstructive apnea-hypopnea index & 19.3 & 15.6 & 21.1 & 6.2 & 0 & 4.5 & 1.1 & 7.7 \\
\hline Central apnea-hypopnea index & 0.2 & 1.1 & 8.2 & 6.2 & 23.2 & 26.9 & 1.8 & 35.7 \\
\hline
\end{tabular}

*inspire is a product of Inspire Medical Systems, Inc., Golden Valley, MN, USA. PAP, positive airway pressure; CPAP, continuous positive airway pressure; BPAP, bilevel positive airway pressure; ASV, adaptive Servo-ventilation 
positive airway pressure (EPAP) from 6 to $14 \mathrm{~cm} \mathrm{H}_{2} \mathrm{O}$. Worsening central respiratory events with ongoing PAP therapy were noted, confirming the diagnosis of TE-CSA (Fig. 1B). Central apneas appeared at a BPAP of $13 / 8$ through BPAP $18 / 14 \mathrm{~cm}$ $\mathrm{H}_{2} \mathrm{O}$. Settings were adjusted multiple times to comfort but the residual central AHI remained high above 20. BPAP-spontaneous/timed (BPAP-ST) was never attempted. Given worsening events on BPAP, ASV titration was recommended.

A second PAP titration with BPAP and adaptive servo-ventilation (ASV) again showed TE-CSA on BPAP with a slight trend towards increased respiratory events in the supine position compared to the lateral position (Fig. 1C). There was significant improvement of TE-CSA after switching from BPAP to ASV after the first half of the study. Obstructive events were controlled at ASV settings with EPAP $6 \mathrm{~cm} \mathrm{H}_{2} \mathrm{O}$, pressure support (PS) maximum of $15 \mathrm{~cm} \mathrm{H}_{2} \mathrm{O}$, PS minimum $3 \mathrm{~cm} \mathrm{H}_{2} \mathrm{O}$, and back up rate was set at auto. Central hypopneas were present in a lower frequency than any other settings, and a recommendation was made for starting ASV. While on ASV therapy, the patient had a residual AHI of 0.8 , and she initially felt better rested. However, only one month after this success, aerophagia returned and PAP therapy had to be stopped a couple of weeks later due to patient intolerance.

She was then fitted for a mandibular advancement device and had a follow-up home sleep study (Resmed Apnea Link Air; Resmed) that revealed inadequate control of respiratory events with 19 obstructive apneas, 120 hypopneas, and 38 central apneas (less than $25 \%$ central events) and an overall respiratory index (RI) of 26.2. Unfortunately, positional data was not available with this portable home sleep study.

Given her intolerance to all PAP therapies and unsuccessful treatment with an oral appliance, the patient was evaluated for HGNS therapy (Inspire, Inspire Medical Systems, Inc., Golden Valley, MN, USA). She was deemed an optimal candidate for HGNS as she met all criteria; she had moderate OSA with PAP intolerance, an initial PSG and follow up portable studies with predominant obstructive events and less than $25 \%$ central events, a BMI less than 32, and drug-induced endoscopy that showed collapse at the level of the oropharynx in an anterior to posterior manner (no complete concentric collapse). After having used CPAP for 72 days, BPAP for 26 days, ASV for 78 days, and a mandibular advancement device, the patient underwent device implantation. Her HGNS titration continued to demonstrate central events with a clear preference for the supine position shown by a supine AHI of 43.4 and a lateral AHI of only 2.9 (Fig. 1D and Table 1). Since a predominance of respiratory events was observed in the supine posture, and adequate control of the patient's apnea was demonstrated with HGNS use during lateral sleep, lateral therapy was implemented. A follow up PSG showed an AHI of 3.3, again demonstrat- ing a positional preference of apneic events with a lateral AHI of 2.0 and a supine AHI of 90.8 , and therefore confirmed treatment success and adequate control of OSA with HGNS and TE-CSA with lateral therapy. During her most recent annual appointment, she described having benefitted from positional therapy, as her poor sleep quality, daytime sleepiness (ESS decreased to 7), total sleep time (increased to 9 hours), and snoring had improved with HGNS after the addition of lateral therapy.

By virtue of this being a single case, our Institutional Review Board policy is that it is exempt by default. Informed consent for publication of this case has been obtained by the patient and is available upon request.

\section{DISCUSSION}

TE-CSA is characterized by central respiratory events that arise or sustain with the use of PAP therapy during treatment of OSA. A reduction of arterial carbon dioxide pressure $\left(\mathrm{PaCO}_{2}\right)$ from a decrease in upper airway resistance, or an increase of arousals in patients who find PAP disruptive, may be responsible for central apneas. ${ }^{2}$ A study of patients with OSA showed a TE-CSA prevalence of $12.2 \%$ that occurred during initial polysomnography with CPAP. ${ }^{3}$ Though TE-CSA may resolve over time with CPAP usage, cases in which central apnea persists can occur. ${ }^{1}$ Devices such as BPAP-ST and ASV are often effective in treating TE-CSA with a backup respiratory rate; ASV is a common treatment for TE-CSA as it affords a backup respiratory rate to support central apnea, and studies have shown ASV's ability to improve the AHI in patients with TE-CSA; ${ }^{4}$ however, apneic events may remain. Unfortunately, our patient developed aerophagia which was likely secondary to PAP therapy and has been repeatedly been described in the literature in patients on PAP therapy. ${ }^{5}$ As muscles relax during sleep, the separation between the airway and the esophagus becomes weaker, allowing air to pass more easily into the esophagus, stomach and sometimes even into the intestines resulting in symptoms like bloating, belching and flatulence.

TE-CSA has previously been described with non-PAP treatments including oral appliances and OSA surgeries. ${ }^{6}$ HGNS is an alternative treatment of OSA in patients who failed PAP therapy. ${ }^{7}$ HGNS decreases the airway resistance and improves apneas and hypopneas which may change the response to chemoreceptors and $\mathrm{PaCO}_{2} \cdot{ }^{6}$ It is possible that these changes can trigger TE-CSA in predisposed patients with sleep instability due to a lower apnea threshold.

Worsening OSA in the supine position is a well-known phenomenon. Positional therapy is a strategy that has been shown to be effective in treating both OSA and central sleep apnea. ${ }^{8,9}$ Interestingly, our patient had no positional preference of her 
OSA on the initial PSG and therefore positional therapy alone would not have been sufficient to control her apneic events in the lateral sleep position. However, she then developed TECSA with PAP therapy with a slight trend towards worsening in the supine position for unclear reasons. Unfortunately, we don't know if the patient had a positional preference of her apneic events while she was using the oral appliance, but she continued to have obstructive and some central events concerning for persistent TE-CSA. The patient finally continued to have TE-CSA with HGNS which occurred almost exclusively in the supine position. The mechanisms that cause central apnea to worsen in the supine position are not entirely clear. ${ }^{9}$ As fluctuating $\mathrm{PaCO}_{2}$ levels during sleep contribute to a normal respiratory drive, variations in these levels can lead to the emergence of disordered breathing. In OSA treated with PAP, relief of obstruction may lead to TE-CSA as the reduction of $\mathrm{PaCO}_{2}$ falls below an apneic threshold. ${ }^{2}$ Variables such as airway shape, lung volume, and loop gain fluctuation have been postulated by Benoist et al. ${ }^{9}$ as probable causes of positional central sleep apnea. Joosten et al. ${ }^{10}$ observed that patients with OSA had an increase in loop gain in the supine versus lateral position. Although it is possible that these components may contribute to positional TE-CSA; research in this area is lacking.

In conclusion, TE-CSA can be present with PAP and nonPAP therapy devices, including HGNS, and in refractory cases positional therapy is a cheap and easy intervention that should be considered. Non-invasive ventilation can assist in treating individuals with TE-CSA, but it is important to keep in mind that these devices may not be effective in certain patients. Our patient's case shows that TE-CSA can persist throughout different treatment modalities, including HGNS, and it may demonstrate a positional preference. The final improvement in our patient's TE-CSA resulted from positional therapy, as respiratory events were still insufficiently controlled with HGNS in the supine position. Positional therapy continues to be invaluable in treating various forms of sleep apnea and may be effective for patients with positional TE-CSA who are refractory to other common therapies and should even be considered more often in patients with central apneas, ideally before using devices like BPAP, BPAP ST or ASV.

\section{Conflicts of Interest}

The authors have no potential conflicts of interest to disclose.
Miriam Jaziri https://orcid.org/0000-0002-6591-8133

Maria Tovar https://orcid.org/0000-0001-6107-6541

\section{Author Contributions}

Conceptualization: Maria Tovar. Data curation: William Palmer, Maria Tovar. Formal analysis: all authors. Investigation: William Palmer, Miriam Jaziri. Resources: Maria Tovar. Supervision: Maria Tovar. Visualization: Miriam Jaziri. Writing_original draft: William Palmer, Miriam Jaziri. Writing_review \& editing: all authors.

\section{Funding Statement}

None.

\section{Acknowledgments}

The authors thank Karla D. Passalacqua, PhD, for assistance with preparation of the manuscript. The authors thank Stephanie Stebens, MLIS, for assistance with preparation of the manuscript.

\section{REFERENCES}

1. Nigam G, Riaz M, Chang ET, Camacho M. Natural history of treatment-emergent central sleep apnea on positive airway pressure: a systematic review. Ann Thorac Med 2018;13:86-91. https://doi.org/10.4103/ atm.ATM_321_17.

2. Berry RB. Fundamentals of sleep medicine. Philadelphia: Saunders, 2012.

3. Cassel W, Canisius S, Becker HF, et al. A prospective polysomnographic study on the evolution of complex sleep apnoea. Eur Respir J 2011;38: 329-337. https://doi.org/10.1183/09031936.00162009.

4. Allam JS, Olson EJ, Gay PC, Morgenthaler TI. Efficacy of adaptive servoventilation in treatment of complex and central sleep apnea syndromes. Chest 2007;132:1839-1846. https://doi.org/10.1378/chest.07-1715.

5. Shepherd K, Hillman D, Eastwood P. Symptoms of aerophagia are common in patients on continuous positive airway pressure therapy and are related to the presence of nighttime gastroesophageal reflux. $J$ Clin Sleep Med 2013;9:13-17. https://doi.org/10.5664/jcsm.2328.

6. Berger M, Solelhac G, Horvath C, Heinzer R, Brill AK. Treatment-emergent central sleep apnea associated with non-positive airway pressure therapies in obstructive sleep apnea patients: a systematic review. Sleep Med Rev 2021;58:101513. https://doi.org/10.1016/j.smrv.2021.101513.

7. Strollo PJ Jr, Soose RJ, Maurer JT, et al. Upper-airway stimulation for obstructive sleep apnea. N Engl J Med 2014;370:139-149. https://doi. org/10.1056/NEJMoa1308659.

8. de Vries GE, Hoekema A, Doff MH, et al. Usage of positional therapy in adults with obstructive sleep apnea. J Clin Sleep Med 2015;11:131137. https://doi.org/10.5664/jcsm.4458.

9. Benoist LBL, Vonk PE, de Vries N, Janssen HCJP, Verbraecken J. Newgeneration positional therapy in patients with positional central sleep apnea. Eur Arch Otorhinolaryngol 2019;276:2611-2619. https://doi. org/10.1007/s00405-019-05545-y.

10. Joosten SA, Landry SA, Sands SA, et al. Dynamic loop gain increases upon adopting the supine body position during sleep in patients with obstructive sleep apnoea. Respirology 2017;22:1662-1669. https://doi. org/10.1111/resp.13108.

\section{ORCID iDs}

William Palmer https://orcid.org/0000-0001-5752-5535 